\title{
Natural and artificial helical structures
}

\author{
F. Carpi ${ }^{1}$, A. Carpi $^{2}$ \& M. A. Russo ${ }^{3,4}$ \\ ${ }^{1}$ Interdepartmental Research Centre 'E. Piaggio', \\ School of Engineering, University of Pisa, Italy \\ ${ }^{2}$ Department of Reproduction and Aging, University of Pisa, Italy \\ ${ }^{3}$ Department of Experimental Medicine, La Sapienza University, Italy \\ ${ }^{4}$ I.R.C.C.S., San Raffaele La Pisana, Italy
}

\begin{abstract}
Helical or coiled structures are very common in several biological materials, such as proteins and nucleic acids. They appear also at a macroscopic level in certain human organs, as in the case of the spiral anatomy of the heart muscle bands or the helical twisting of the umbilical cord. Further examples can be found in the rest of the natural world, such as in the structure of certain trees or even in the agglomeration of galactic nebulae and in plasma jets of quasars. Beyond the biological and natural domains, artificial helical structures from the nano- to the macro-scale have been developed by science and technology. Nanosprings made of zinc oxide, helical microtubules of graphitic carbon, helical screws and gears, and the helical flying machine dreamed about by Leonardo da Vinci are just a few outputs of the human interest for this shape. This paper intends to provide a brief overview on natural and artificial examples of helical structures, showing how their geometrical properties have been exploited to achieve different purposes.
\end{abstract}

Keywords: artificial, helical, helix, natural, structure.

\section{Introduction}

The natural world has always significantly attracted human interest for the variety of examples of fascinating regularity and symmetry of which it is capable. Both living bodies and inanimate structures showing incredible geometrical properties permeate our world, spanning from infinitesimal domains to astronomical scales. 
Helical or coiled structures are one of the most significant examples of such an attractive feature of nature. They are very common in several biological materials, such as proteins and nucleic acids. They can be found even in certain human organs, as well as in the rest of the natural world.

Of course, nature is not the exclusive 'provider' and 'user' of helical structures. Beyond the biological and natural domains, in fact, artificial helical structures have been exploited by men during centuries of scientific and technological development. As a consequence, different types of products based on helical shapes surround us, ranging from the nano- to the macro- scale.

This paper intends to provide just a brief overview on some of the most significant examples of natural and artificial helical structures, showing how their geometrical properties are exploited to achieve different purposes.

\section{Natural helical structures}

In 1953, the double helix model of DNA (deoxyribonucleic acid) was described by Watson and Crick [1]. Since then, the double helical structure of DNA (fig. 1) has become the symbol of molecular biology.

The peculiarity of this structure suggests its role as a copying mechanism for the incredibly huge amount of genetic information, which is stored by DNA and is used to assemble proteins.

Proteins are biopolymers consisting amino-acids linked together into chains, by means of the so-called peptide bond (polypeptides). Such chains of aminoacids are common in the form of a helix [3].

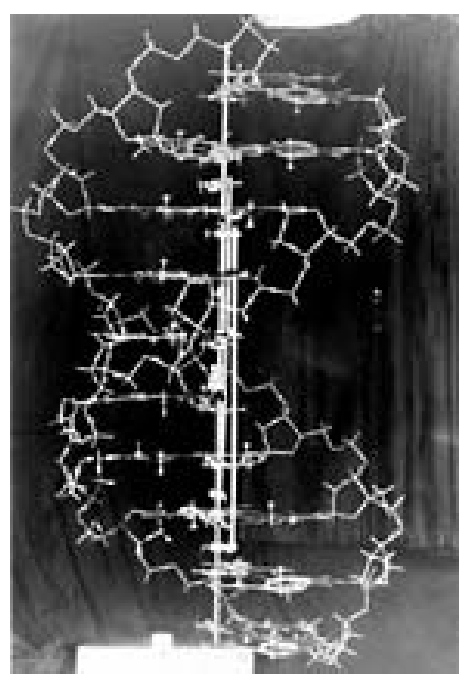

Figure 1: Original stick-and-ball model of DNA by Watson and Crick (source: Cold Spring Harbor Laboratory Archives; from [2]). 


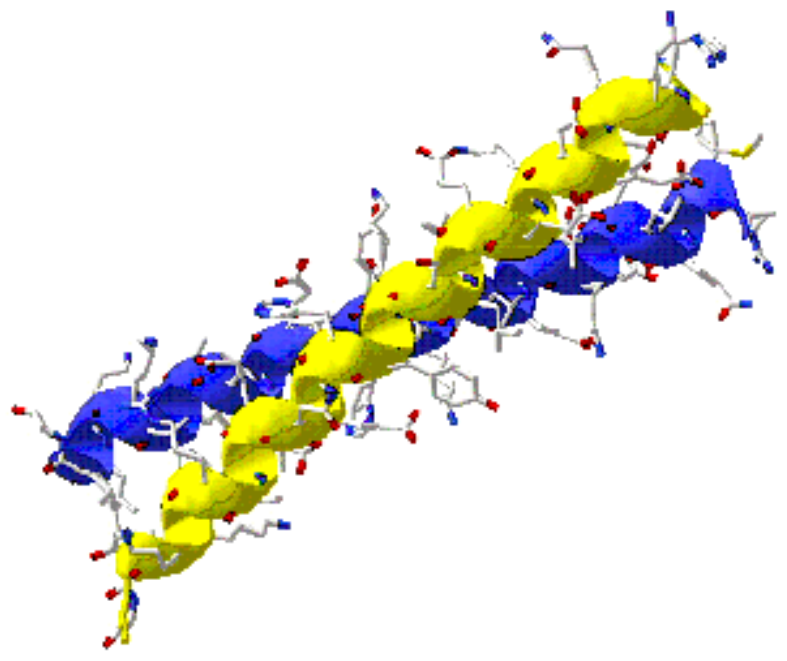

Figure 2: $\quad$ Schematic drawing of an example of $\alpha$-helical proteins.

For instance, this is the case of the so-called $\alpha$-helical membrane proteins (fig. 2). Such proteins can be found within the membrane of cells. In fact, they usually form cell-membrane channels. As an example, it has been shown how the helical structure of the proteins which constitute potassium channels is used to drive potassium ions into the cell. In particular, various stretches of aminoacids within the channel coil up into helical structures, whose distributed electrical charges draw positively charged ions toward the inside of the cell [4]. Several other examples of $\alpha$-helical membrane proteins can be easily identified. For instance, some of them are listed in [5].

In addition to the functional relevance of the helical structure for transport properties of membrane proteins, studies have demonstrated even the considerable importance of helical packing for folding, stability and association of such proteins [6]. As an example, some bacteria can survive in boiling water due to the fact that their natural polypeptides are particularly stabilized by a helical structure.

The $\alpha$-helical structure can be found even in different kinds of proteins, such as the fibrous-like ones. Examples in this respect include collagen, actin, myosin and tropomyosin.

Collagen is a protein of connective tissue. It presents a rod-like structure, made of three helical polypeptide chains (triple helix) twisted around each other [7]. Each chain contains about one thousand of amino-acids. Collagen molecules align to form microfibrils and microfibrils align to form collagen fibers. The triple helix solution seems to have been adopted by nature in order to achieve a sort of fiber-reinforced composite structure, which provides connective tissues with adequate structural strengths. 
Fibrils integrating $\alpha$-helical structures are found in muscles too. They are myofibrils, which originate from the alignment of contractile proteins: actin and myosin. Each of them consists of a rod, made of two chains of $\alpha$-helical coiled coils (double helix). In this case, the helical structure is functional to the interaction to be established between the two myosin 'heads' and actin in order to generate the contractile force [8].

A couple of additional examples are able to further demonstrate advantages offered by helical structures for biological contraction phenomena. One is related to muscular fibers in worms. They present a helical arrangement around the animal tubular body. This permits them to resist an internal pressure, while turning it into tension in the fibers [9]. As a second example of involvement of helical structures in biological contractions, let's refer to the heart. It is well known that the myocardial muscular bands show a helical spatial arrangement, as depicted in fig. 3 [10-12].

Such an arrangement is likely functional to ventricular performance. In fact, ventricular activation has been shown to follow the helical trajectory of the myocardial bands [10].

Another human structure shows macroscopic evidences of the use of the helical shape. It is the fetal umbilical cord. It is a conduit of blood vessels which extends from the umbilicus of the fetus to the fetal surface of the placenta. Normal human umbilical cords have two small arteries and one large vein. Typically, the two arteries are spiraled around the vein, running a helical course through the umbilical cord [13-15], as shown in fig. 4.

It has been reported that the helical nature of the cord is possibly controlled by factors which may be partly genetic and partly environmental [15]. However, this configuration can vary, implying alterations to the blood supply. For instance, in certain cases, the vein can also parallel the arteries in a helical configuration, or the vein can wind around the arteries. Definitely, the helical arrangement of these vessels plays an important role for the blood supply to the fetus.

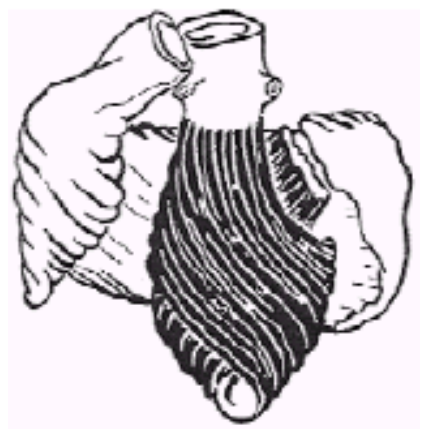

Figure 3: Helical configuration of ventricular myocardial bands (adapted from [10]). 


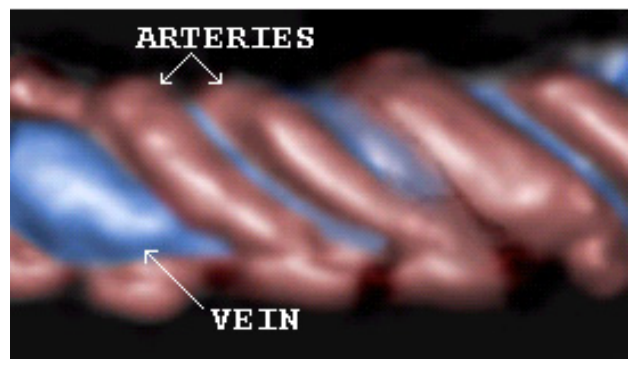

Figure 4: Color-coded 3D Doppler image showing blood flow through a human umbilical cord (adapted from [16]).

Several additional examples of natural helical or spiral structures may be mentioned, such as the human cochlea, the horns of some animals, the shape of sea shells or even the agglomeration of galactic nebulae and quasars. Nevertheless, due to the necessary limitation of this brief survey, it is here useful to have a glance also to examples of artificial helical structures, as reported in the following section.

\section{Artificial helical structures}

Beyond the biological and natural domains, the peculiar properties of helical shapes have largely been exploited by the human technological progress. Nowadays, artificial helical structures surround us in many different types of environments and objects. Their adoption in mechanical practice provided the technological progress with a revolutionary boost.

It is likely that some natural examples of macroscopic helical structures, such as the shape of certain shells, animal horns or trees, may have inspired the development of the first artificial helical structures. However, it's definitely hard to try to guess how much (if ever) such natural examples may have driven the actual development of the first artificial structures. This is particularly evident in consideration of the limited diffusion of information available to humans at the time they introduced the helical technology. Definitely, experimental practice and intuition should have played a fundamental role too.

The worm screw has been certainly one of the most relevant inventions of the early technological era. This gearing mechanism revolutionized the mechanical world. One of the first fundamental inventions was the Archimedes' water screw: a very ingenious solution to carry and 'lift' water from a lower to an upper level (fig. 5).

Screws provided a new and useful means to transfer motion to several kinds of structures, such as slides, carriages or sluice gates.

Leonardo da Vinci conceived one of his flying machines as based on a helical structure, the so-called 'aerial screw, known also as 'helicopter'. The machine was thought to be equipped with a large piece of linen arranged as a helical screw (fig. 6). 


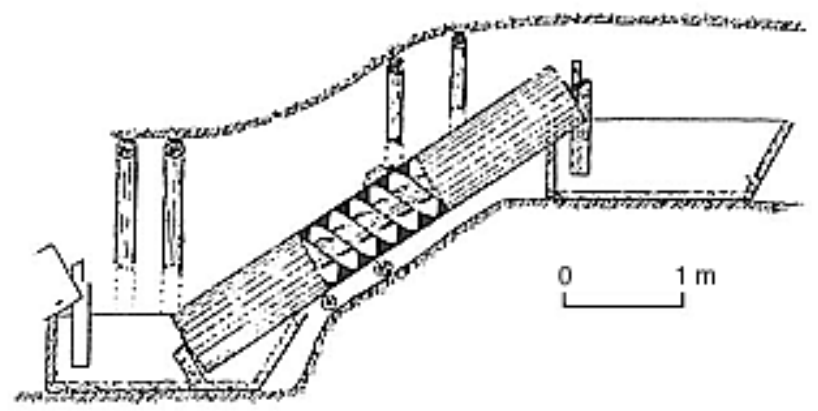

Figure 5: $\quad$ Archimedes' water screw (from [17]).

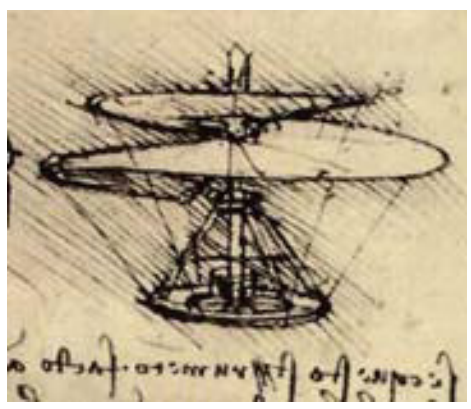

Figure 6: Leonardo's aerial screw (drawing Ms. B, f. 83v).

In particular, the machine design was conceived as a spiral of linen around a vertical shaft. Although this machine did never fly (the genius of Leonardo had to face for several inventions with the irreparable lack of adequate power sources), it demonstrated the great intuition of this inventor about the potential aerodynamic properties of helical shapes.

Of course, there's no need to underline how screws are so largely exploited today.

In addition to the large use of rigid helical structures, a comparable number of applications exploit today elastic helical structures (springs). They are diffusely adopted for mechanical energy accumulation and damping. They can be found in a huge number of products, ranging from mechanical wristwatches to motor vehicles.

Helical structures are nowadays invading nanotechnology as well. They may find at nano-scale applications as components for nanodevices. As an example, a technique to obtain nanohelices of zinc oxide $(\mathrm{ZnO})$ was recently described $[18,19]$. Helices with diameters of $300-700 \mathrm{~nm}$, pitches of $500-2500 \mathrm{~nm}$ and lengths up to $100 \mu \mathrm{m}$ were obtained, as shown in Fig. 7 [18].

Owing to the semiconductive and piezoelectric properties of $\mathrm{ZnO}$, such nanosprings may result useful for nano-scale electromechanics and optoelectronics as sensors, actuators and resonators. 


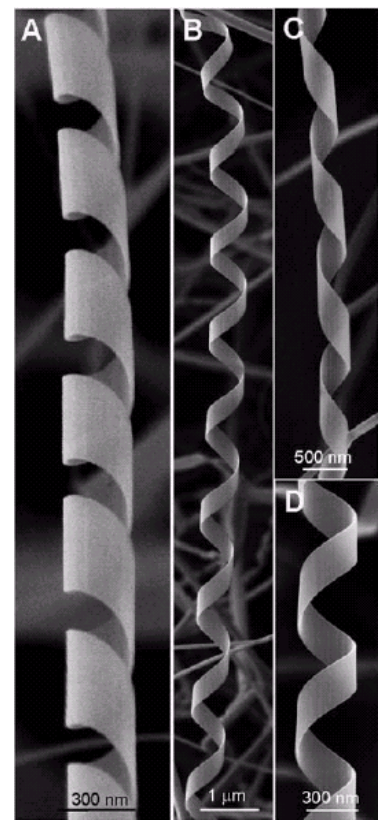

Figure 7: $\quad$ SEM images of $\mathrm{ZnO}$ nanohelices (from [18]).

\section{Conclusions}

This paper has briefly highlighted some natural and artificial examples of helical structures. Evolution has exploited this geometrical shape to provide living creatures with different functions, enabled, at various scales, by helical features. Similarly, human technology has learned to develop and use helical structures from the macro- to the nano-scale, in order to strengthen its attempts of ruling his world. Such examples coming from both natural and artificial domains demonstrate the relevance of helical structures for our world and our own lives.

\section{References}

[1] Watson, J.D. and Crick, F.H.C., A Structure for deoxyribose nucleic acid, Nature 171, pp. 737-738, 1953.

[2] Web site: http://www.ornl.gov/sci/techresources/Human_Genome/publicat/ primer2001/1.shtml

[3] Cohen, C. and Perry, D.A.D., $\alpha$-helical coiled coils and bundles: How to design an $\alpha$-helical protein, Proteins: Structure, Function, and Genetics 7, pp. 1-15, 1990.

[4] Travis, J., Channel Surfing, Science News 161(10), p. 152, 2002.

[5] Eyre, T.A., Partridge, L. and Thornton, J.M., Computational analysis of $\alpha-$ helical membrane protein structure: implications for the prediction of $3 \mathrm{D}$ structural models, Protein Eng. Des. Sel. 17, pp. 613-624, 2004. 
[6] Eilers, M., Shekar, S.C., Shieh, T., Smith, S.O., and Fleming, P.J., Internal packing of helical membrane proteins, PNAS 97(11), pp. 5796-5801, 2000.

[7] Lehninger, L., Nelson, D.L., Cox, M.M., Principles of Biochemistry, Worth: New York, 1993.

[8] Li, Y., Brown et al., Visualization of an unstable coiled coil from the scallop myosin rod, Nature 424, pp. 341-45, 2003.

[9] Alexander, R. McN., Animal Mechanics, Oxford: Blackwell Scientific Publications, 1983.

[10] Torrent-Guasp, F. et al., Spatial orientation of the ventricular muscle band: physiologic contribution and surgical implications, J. Thorac. Cardiovasc. Surg. 122(2), pp. 389-92, 2001.

[11] Buckberg, G., Basic science review: the helix and the heart, J. Thorac. Cardiovasc. Surg., 124(5), pp. 863-883, 2002.

[12] Marino B. and Corno A.F., Spiral pattern: universe, normal heart, and complex congenital defects, J. Thorac. Cardiovasc. Surg. 126(4), pp. 12251226, 2003.

[13] Predanic, M. et al., Assessment of umbilical cord coiling during the routine fetal sonographic anatomic survey in the second trimester, $J$ Ultrasound Med 24, pp. 185-191, 2005.

[14] Qin, Y., Lau, T.K. and Rogers, M.S., Second-trimester ultrasonographic assessment of the umbilical coiling index, Ultrasound in Obstetrics \& Gynecology, 20(5), p. 458, 2002.

[15] Chaurasia, B.D., Agarwal, B.M., Helical structure of the human umbilical cord, Acta Anat (Basel), 103(2), pp. 226-230, 1979.

[16] Web site: http://www.amnionet.com/cord.htm.

[17] Web site: http://www.math.nyu.edu/ crorres/Archimedes/Screw/ SourcesScrew.html

[18] Gao, P.X. et al., Conversion of zinc oxide nanobelts into superlatticestructured nanohelices, Science 309, pp. 1700-1704, 2005. 